\title{
Synthesis of functionalized thiophene/phenyl co-oligomers by direct arylation of thiophenes
}

\author{
Andreas Osadnik and Arne Lützen* \\ University of Bonn, Kekulé-Institute of Organic Chemistry and Biochemistry, \\ Gerhard-Domagk-Str. 1, D-53121 Bonn, Germany \\ E-mail: arne.luetzen@uni-bonn.de
}

Dedicated to Prof Dr. Jürgen Martens

DOI: http://dx.doi.org/10.3998/ark.5550190.p008.833

\begin{abstract}
$\pi$-Conjugated rod-like molecules have proven to be powerful candidates for the generation of well-defined nanostructures with interesting optical and optoelectrical properties. Their synthesis, however, turns out to be rather complex in some cases. Here, we have optimized the synthesis of oligomers consisting of thiophene and phenylene groups with three or four aromatic units using a direct arylation approach, which allows us to use cheap starting materials and to minimize the number of reaction steps considerably.
\end{abstract}

Keywords: Thiophenylphenylenes, direct arylation, $\pi$-conjugated oligomers, cross-coupling reactions, thiophene

\section{Introduction}

The use of rod-like $\pi$-conjugated organic oligomers ${ }^{1,2}$ in the synthesis of organic materials has witnessed a tremendous development recently due to their interesting optical, electrical and optoelectrical properties and their high tendency towards self-aggregation processes. ${ }^{3-12}$

Among these, para,para'-functionalized oligophenylenes bearing various substituents have been found to self-assemble into well-defined, mutually aligned fiber-like nanostructures upon vapor deposition onto a freshly cleaved mica surface. ${ }^{13}$ Changing the molecules' chain length and substitution pattern does not only have an effect on the electrical and optical properties on the molecular level, but also on the self-aggregation and the physical properties of the nanostructures. ${ }^{14,15}$ However, this approach asks for oligomers of four or more aromatic units in order to have a vapor-pressure in the right regime to allow sublimation under ultra-high vacuum 
conditions $\left(\leq 10^{-7}\right.$ mbar) whereas oligomers containing only three aryl units are usually too volatile under these conditions.

Another well-established way to obtain nano-structured surfaces is the use of porous templates. ${ }^{16}$ Here, the organic molecules are deposited from solution in the pores of an alumina template. After selectively dissolving the alumina layer, the surface is covered with upstanding nanowires. An important requirement for this procedure is an adequate solubility of the used molecules. Non-substituted or only para,para'-substituted quaterphenylenes or even longer oligomers hardly fulfill this requirement since they are barely soluble. The synthesis of hybrid compounds containing different kinds of aromatic rings is a common approach to fine-tune the chemical and physical properties of $\pi$-conjugated molecules. ${ }^{2-12}$ In this respect, the combination of phenylenes and thiophenes has proven to be very successful in order to access optoelectronic devices with improved properties. ${ }^{17-28}$ Compounds that are exclusively functionalized in position 4 of a terminal phenyl group and/or in position 2 of a terminal thiophene unit, however, are fairly rare, although they are also very promising candidates for the formation of nanoaggregates. Therefore, we started to develop a general approach for the synthesis of thiophenyl-phenylenes hybrid oligomers containing a total of three aromatic units and (at least) one biphenyl group as a reasonable compromise between interesting optical and optoelectronical properties on the one hand and solubility on the other hand.

\section{Results and Discussion}

A direct functionalization of the parent structure, 2-(biphenyl-4-yl)thiophene, is rather difficult, especially with regard to regioselectivity in the phenylene part. Therefore, it is wiser to introduce the functional groups into suitable precursors before the desired $\pi$-conjugated oligomer skeleton is formed, e.g. via transition metal cross-coupling approaches. ${ }^{29}$ Especially the Suzuki-, Kumada-, and Stille-reaction have been successfully employed in this context. ${ }^{30-34}$ Following this approach, however, asks for the synthesis of either a 4'-substituted 4-iodobiphenyl and a 2functionalized thiophene-5-yl boronic acid or stannyl derivative or vice versa. Depending on the nature of the additional substituents this might be quite tedious. Nevertheless, we followed this approach but the results of some Suzuki-cross coupling reactions were quite unsatisfactory in our hands despite various optimization steps. In fact, only one compound could be synthesized in a satisfying yield of 50\% using a Buchwald-ligand ${ }^{35}$ (Scheme 1).

Hence, we decided to explore the potential of direct arylation ${ }^{36,37}$ as the key step to establish the desired $\pi$-conjugated oligomer skeleton. In fact, this turned out to be very successful since we could synthesize a vast number of different compounds from cheap starting materials in only a few reaction steps.

Our route started with the synthesis of 4'-substituted 4-iodobiphenyls from 4-iodoaniline. Therefore, we followed a three-step protocol that we reported earlier (Scheme 2). ${ }^{38,39}$ 


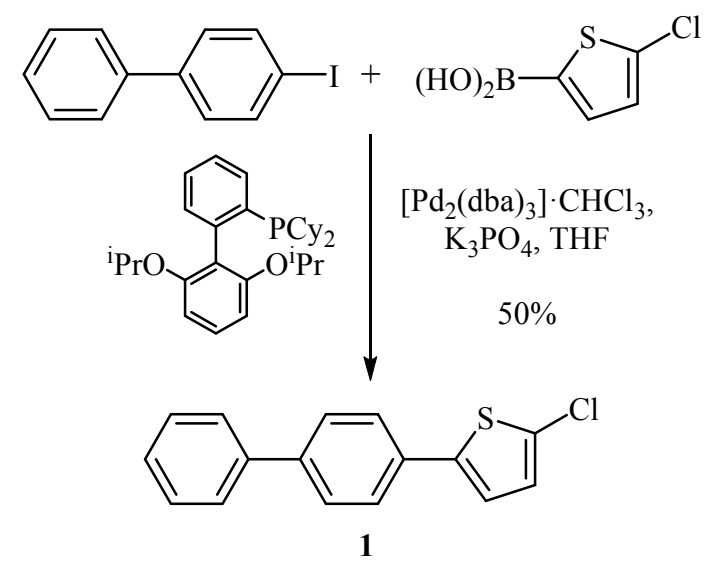

Scheme 1 Synthesis of 2-(biphenyl-4-yl)-5-chlorothiophene
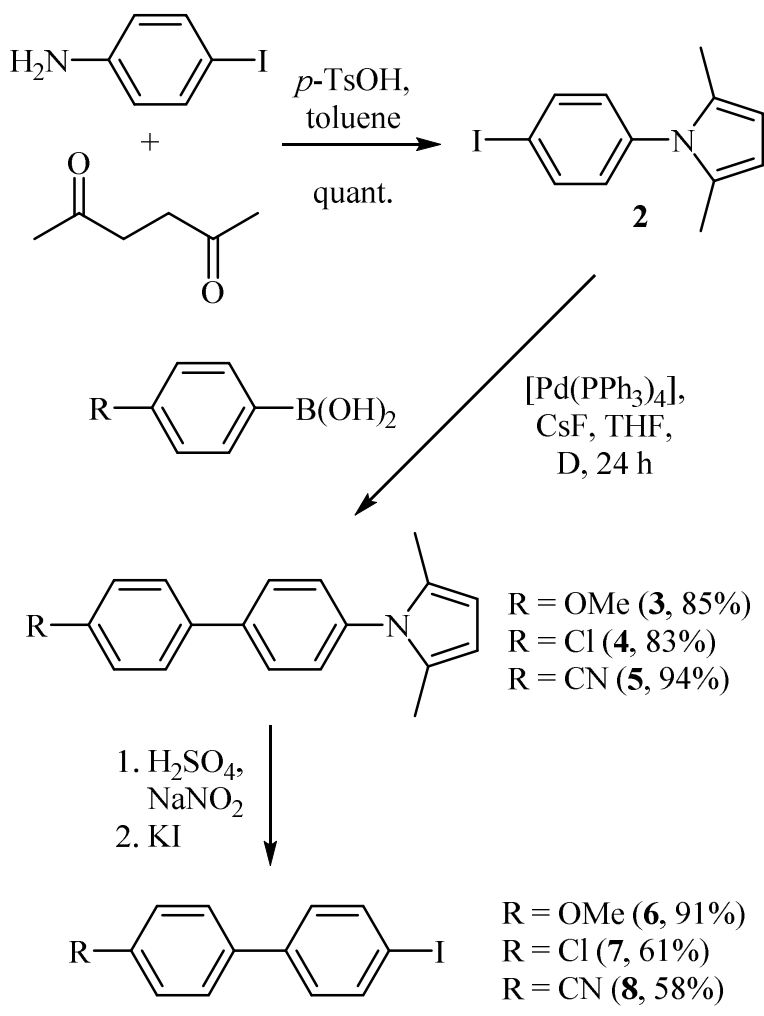

Scheme 2 Synthesis of 4' functionalized 4'-iodobiphenyls 6-8 from 4-iodoaniline

These 4'-functionalized-4-iodobiphenyls were subsequently used in direct arylation reactions with 2 -substituted thiophenes. In the literature, some synthetic approaches using Heck-reactions in the cross-coupling of functionalized thiophene-rings are described. Lavenot et al., ${ }^{40}$ e.g., described a protocol for Heck-reactions that they could apply for the direct arylation of activated thiophenes, using classical reaction conditions (palladium(II) acetate and tetrabutylammonium 
bromide). ${ }^{41}$ Adopting this protocol for our synthetic approach, we were able to synthesize 2(biphenyl-4-yl)-5-cyanothiophene, but the reaction yield turned out to be unsatisfactory. Thus, we tried to find a better alternative.

In a next step we tested a protocol reported by A. Mori et al. ${ }^{42-44}$ who were able to couple 2bromothiophenes with aryl iodides: the 4'-functionalized 4-iodobiphenyls were reacted with 2functionalized thiophenes using 5 mol-\% $\left[\mathrm{PdCl}_{2}\left(\mathrm{PPh}_{3}\right)_{2}\right], \mathrm{PPh}_{3}$, potassium fluoride, and silver nitrate as the catalytic system to obtain the thiophenylphenylenes in moderate to acceptable yields of 58\% (9) and 33\% (10) (Scheme 3). Whereas the solubility of 9 is sufficient for purification via column chromatography, $\mathbf{1 0}$ was purified by filtering the precipitated product and repeatedly washing with various solvents.

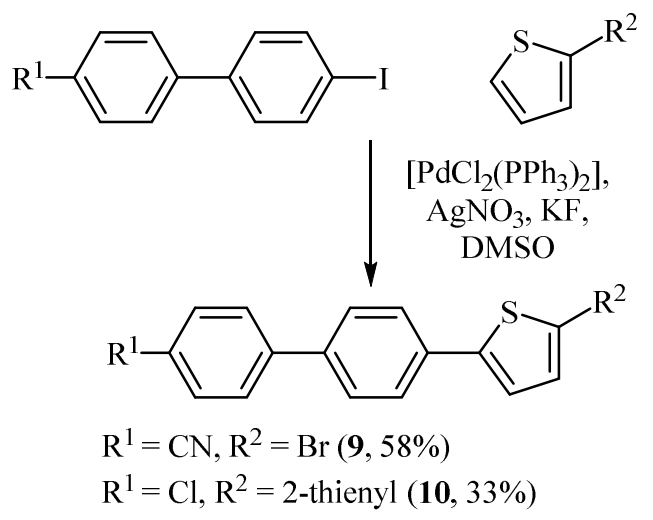

Scheme 3 Synthesis of 2-thienylphenylenes according to a protocol by A. Mori

However, the synthesis of compounds with other substituents than a bromine atom or a 2thienyl group still turned out to be either unsatisfactory or impossible. Therefore, we also tested conditions published by Greaney et al. who reported about the successful use of $\left[\mathrm{Pd}(\mathrm{dppf}) \mathrm{Cl}_{2}\right]$, $\mathrm{PPh}_{3}$, and $\mathrm{Ag}_{2} \mathrm{CO}_{3}$ in the arylation of thiazoles. ${ }^{45}$ Adopting this strategy for our synthetic purpose, we were able to synthesize several thiophenylphenylenes with good to excellent yields (Scheme 4). However, it is important to note that we had to extend the reaction time up to seven days to obtain satisfying yields. 


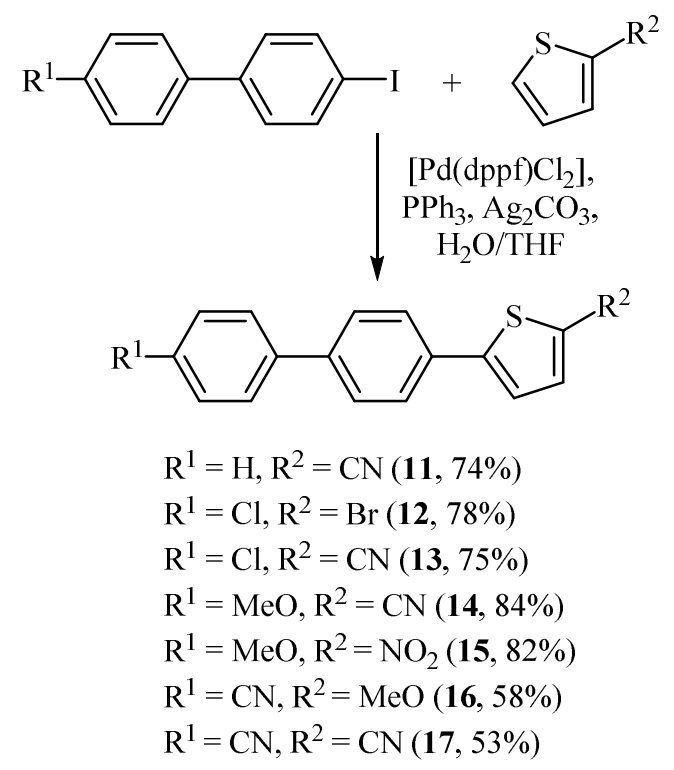

Scheme 4 Synthesis of 2-thienylphenylenes according to a protocol by M. F. Greaney et al.

\section{Conclusions}

In conclusion, we have developed two reliable protocols for the synthesis of nine functionalized 2-thienylphenylenes with three or four aromatic units and a broad variety of functional groups by employing a Heck-reaction to establish the $\pi$-conjugated oligomeric backbone. All reactions were optimized with regard to reaction yields. In all cases, we were able to isolate the desired compound in sufficient purity. All compounds have interesting optical and optoelectronical properties and are promising candidates for the formation of nanostructures via a templatemethod which will be examined in due course.

\section{Experimental Section}

General. Solvents were dried, distilled and stored under argon according to standard procedures. Reactions with air- and moisture-sensitive transition-metal compounds were performed under an argon atmosphere in oven-dried glassware using standard Schlenk techniques.

Thin-layer chromatography was performed on aluminum TLC plates (silica gel 60) from Merck and the products were visualized under UV-light $(254$ or $366 \mathrm{~nm})$. Products were purified by column chromatography on silica gel 60 (70-230 mesh or 230-400 mesh) from Merck.

${ }^{1} \mathrm{H}-\mathrm{NMR}$ and ${ }^{13} \mathrm{C}-\mathrm{NMR}$ spectra were recorded at $298 \mathrm{~K}$ on a Bruker AM 400 spectrometer operating at $400.1 \mathrm{MHz}\left({ }^{1} \mathrm{H}\right)$ and $100.6 \mathrm{MHz}\left({ }^{13} \mathrm{C}\right)$, respectively. Mass spectra were recorded on a Finnigan MAT-95XL. UV/Vis-spectra were measured on a Jena Analytic Specord 200 
spectrometer in a $1 \mathrm{~cm}$ quartz cuvette. Elemental analyses were carried out with a Heraeus Vario EL instrument. Since the products were obtained as amorphous solids, they usually contain residual amounts of solvents from the extraction and washing procedure that could not be removed completely in all cases even after heating for longer periods of time under standard laboratory vacuum $\left(10^{-3}\right.$ mbar). We give HRMS data in all cases.

4-Iodoaniline, 4-methoxyphenylboronic acid, 4-chlorophenylboronic acid, 4-cyanophenylboronic acid, 2-bromothiophene, 5-chlorothiophen-2-ylboronic acid, 4-iodobiphenyl, 2dicyclohexylphosphino-2',6'-diisopropoxybiphenyl, 2-cyanothiophene, 2-methoxy-thiophene, 2nitrothiophene, 2,2'-bithiophene, $\mathrm{K}_{3} \mathrm{PO}_{4}, \mathrm{KF}, \mathrm{CsF}, \mathrm{AgNO}_{3}, \mathrm{PPh}_{3}, \mathrm{NaNO}_{2}, \mathrm{Ag}_{2} \mathrm{CO}_{3}$, $\left[\mathrm{PdCl}_{2}\left(\mathrm{PPh}_{3}\right)_{2}\right],\left[\mathrm{Pd}_{2}(\mathrm{dba})_{3}\right] \cdot \mathrm{CHCl}_{3},\left[\mathrm{Pd}(\mathrm{dppf}) \mathrm{Cl}_{2}\right]$, and $\left[\mathrm{Pd}\left(\mathrm{PPh}_{3}\right)_{4}\right]$ were purchased from SigmaAldrich, Alfa Aesar, ABCR, or Acros Organics and used as received.

1-(2,5-Dimethyl-1H-pyrrol-1-yl)-4-iodobenzene (2), ${ }^{46}$ 4-(2,5-dimethyl-1H-pyrrol-1-yl)-4'methoxybiphenyl (3), ${ }^{38}$ 4-chloro-4'-(2,5-dimethyl-1H-pyrrol-1-yl)biphenyl (4), ${ }^{38}$ 4-cyano-4'(2,5-dimethyl-1H-pyrrol-1-yl)biphenyl (5), ${ }^{38}$ 4-iodo-4'-methoxybiphenyl (6), ${ }^{38}$ 4-chloro-4'iodobiphenyl (7), ${ }^{38}$ and 4 -cyano-4'-iodobiphenyl ${ }^{38}$ were prepared according to published procedures.

2-(Biphenyl-4-yl)-5-chlorothiophene (1). A two-neck flask equipped with a condenser was charged with 5-chlorothiophene-2-ylboronic acid (150 mg, $0.93 \mathrm{mmol}, 1.05$ equiv.), 4iodobiphenyl (250 mg, $0.88 \mathrm{mmol}, 1$ equiv.), $\mathrm{K}_{3} \mathrm{PO}_{4}$ (4.86 g, $5.28 \mathrm{mmol}, 6$ equiv.), $\left(\left[\mathrm{Pd}_{2}(\mathrm{dba})_{3}\right] \cdot \mathrm{CHCl}_{3} \quad(28 \mathrm{mg}, 0.03 \mathrm{mmol}, 3 \mathrm{~mol}-\%)\right.$, and 2-dicyclohexylphosphino-2',6'diisopropoxybiphenyl (25 mg, $0.05 \mathrm{mmol}, 5 \mathrm{~mol}-\%)$. THF (30 mL) was added and the reaction mixture was heated to reflux for $24 \mathrm{~h}$. After cooling down to room temperature, the solution was extracted with $\mathrm{CH}_{2} \mathrm{Cl}_{2}$ repeatedly. The combined organic phases were washed with $\mathrm{H}_{2} \mathrm{O}(2 \times 20$ $\mathrm{mL})$ and dried $\left(\mathrm{Na}_{2} \mathrm{SO}_{4}\right)$. The solvent was removed under reduced pressure and the crude product was purified by column chromatography on silica gel using petroleum ether/ethyl acetate (30:1 $\mathrm{v} / \mathrm{v})$ as eluent to give the desired product as a yellow amorphous solid. Yield: $130 \mathrm{mg}(50 \%)$.

${ }^{1} \mathrm{H}-\mathrm{NMR}\left(400 \mathrm{MHz}, \mathrm{CDCl}_{3}\right.$ ): $\delta$ 7.55-7.65 (m, $\left.6 \mathrm{H}\right)$, 7.43-7.50 (m, $\left.2 \mathrm{H}\right), 7.34-7.40(\mathrm{~m}, 1 \mathrm{H}, J$ 9.0 $\mathrm{Hz}), 7.11(\mathrm{~d}, 1 \mathrm{H}, J 3.9 \mathrm{~Hz}), 6.91(\mathrm{~d}, 1 \mathrm{H}, J 3.9 \mathrm{~Hz}) \mathrm{ppm} .{ }^{13} \mathrm{C}-\mathrm{NMR}\left(100 \mathrm{MHz}, \mathrm{CDCl}_{3}\right): \delta 142.7$, $140.8,140.5,132.8,129.3,129.0,127.8,127.7,127.3,127.1,126.0,122.4$ ppm. MS (EI): $\mathrm{m} / z$ (\%) 270.0 (100) [ $\mathrm{M}^{\circ+}$ ]. HRMS (EI): $\mathrm{m} / z$ calcd for $\mathrm{C}_{16} \mathrm{H}_{11}{ }^{35} \mathrm{ClS}: 270.0270$; found: 270.0267 . Anal. calcd for $\mathrm{C}_{16} \mathrm{H}_{11} \mathrm{ClS}$ : C: 70.97; H: 4.09; S:11.84; found: $\mathrm{C}: 70.74 ; \mathrm{H}: 4.24$; S: 11.78. UV/Vis $\left(\mathrm{CH}_{2} \mathrm{Cl}_{2}\right): \lambda_{\max } 232 \mathrm{~nm}$. Fluorescence $\left(\mathrm{CH}_{2} \mathrm{Cl}_{2}\right): \lambda_{\max } 376 \mathrm{~nm}$.

Synthesis of Oligothiophenes; General Procedure 1 (GP 1). A two-neck flask equipped with a condenser was charged with a 4'-substituted-4-iodobiphenyl (1 equiv.), potassium fluoride (2 equiv.), $\left[\mathrm{PdCl}_{2}\left(\mathrm{PPh}_{3}\right)_{2}\right]$, and DMSO $(15 \mathrm{~mL})$. Under an argon atmosphere a 2-substituted thiophene (1.2 equiv.) was added. Silver nitrate (1 equiv.) was then added in one portion. The reaction mixture was stirred for $24 \mathrm{~h}$ at $100{ }^{\circ} \mathrm{C}$. After cooling down to room temperature, the solution was filtered through a Celite pad, which was washed with $\mathrm{CH}_{2} \mathrm{Cl}_{2}$ repeatedly. The filtrate was washed with $\mathrm{H}_{2} \mathrm{O}(2 \times 40 \mathrm{~mL})$, dried over $\mathrm{MgSO}_{4}$, and the solvent was removed 
under reduced pressure. The crude product was purified by column chromatography on silica gel to give the desired product.

Synthesis of Oligothiophenes; General Procedure 2 (GP 2). A two-neck flask equipped with a condenser was charged with silver carbonate (2 equiv.), [Pd(dppf) $\left.\mathrm{Cl}_{2}\right](5 \mathrm{~mol}-\%), \mathrm{PPh}_{3}\left(10 \mathrm{~mol}^{-}\right.$ $\%$ ), a 4'-substituted-4-iodobiphenyl (1.2 equiv.), and a 1:1 mixture of $\mathrm{H}_{2} \mathrm{O} / \mathrm{THF}$. Under an argon atmosphere, a 2-substituted thiophene (1 equiv.) was added and the reaction mixture was stirred for $7 \mathrm{~d}$ at $60{ }^{\circ} \mathrm{C}$. After cooling down to room temperature, the solution was filtered through a Celite pad, which was washed with acetone and $\mathrm{CH}_{2} \mathrm{Cl}_{2}$ repeatedly. The filtrate was concentrated and brine and $\mathrm{CH}_{2} \mathrm{Cl}_{2}\left(5 \mathrm{~mL}\right.$ each) were added. The organic phase was dried $\left(\mathrm{MgSO}_{4}\right)$ and the solvent was removed under reduced pressure. The crude product was purified by column chromatography on silica gel to give the desired product.

4'-(5-Bromo-2-thienyl)-[1,1'-biphenyl]-4-carbonitrile (9). 4-Cyano-4'-iodobiphenyl (300 mg, $0.98 \mathrm{mmol})$, potassium fluoride $(114 \mathrm{mg}, 1.96 \mathrm{mmol}),\left[\mathrm{PdCl}_{2}\left(\mathrm{PPh}_{3}\right)_{2}\right](35 \mathrm{mg}, 0.05 \mathrm{mmol}), 2-$ bromo-thiophene ( $1.18 \mathrm{mmol}, 0.12 \mathrm{~mL}, 1.2$ equiv.), and silver nitrate (167 mg, $0.98 \mathrm{mmol}$ ) were reacted in DMSO $(15 \mathrm{~mL})$ according to GP1 to give the desired product as a yellow amorphous solid after purification by column chromatography on silica gel using petroleum ether/ethyl acetate $(9: 1 \mathrm{v} / \mathrm{v})$ as eluent. Yield: $192 \mathrm{mg}(58 \%) .{ }^{1} \mathrm{H}-\mathrm{NMR}\left(400 \mathrm{MHz}, \mathrm{CDCl}_{3}\right): \delta 7.73(\mathrm{~d}, 2 \mathrm{H}, J$ $8.7 \mathrm{~Hz}), 7.68$ (d, $2 \mathrm{H}, J 8.7 \mathrm{~Hz}), 7.61$ (d, $2 \mathrm{H}, J 9.0 \mathrm{~Hz}), 7.59$ (d, $2 \mathrm{H}, J 8.9 \mathrm{~Hz}), 7.12$ (d, $1 \mathrm{H}, J$ $3.9 \mathrm{~Hz}), 7.05$ (d, $1 \mathrm{H}, J 3.9 \mathrm{~Hz}) \mathrm{ppm} .{ }^{13} \mathrm{C}-\mathrm{NMR}\left(100 \mathrm{MHz}, \mathrm{CDCl}_{3}\right): \delta 145.0,144.8,138.5,134.1$, 132.8, 131.2, 128.0, 127.6, 126.3, 123.9, 119.0, 112.3, 111.2 ppm. MS (EI): $m / z(\%) 341.0(100)$ $\left[\mathrm{M}^{\bullet+}\right.$ ]. HRMS (EI): $\mathrm{m} / z$ calcd for $\mathrm{C}_{17} \mathrm{H}_{10}{ }^{79} \mathrm{BrNS}$ : 338.9717; found: 338.9715 . UV/Vis $\left(\mathrm{CH}_{2} \mathrm{Cl}_{2}\right)$ : $\lambda_{\max } 234 \mathrm{~nm}$. Fluorescence $\left(\mathrm{CH}_{2} \mathrm{Cl}_{2}\right): \lambda_{\max } 408 \mathrm{~nm}$

5-(4'-Chlorobiphenyl-4-yl)-2,2'-bithiophene (10). 4-Chloro-4'-iodobiphenyl (300 mg, $0.95 \mathrm{mmol}$ ), potassium fluoride (111 mg, $1.91 \mathrm{mmol}),\left[\mathrm{PdCl}_{2}\left(\mathrm{PPh}_{3}\right)_{2}\right]$ (34 mg, $\left.0.05 \mathrm{mmol}\right), 2,2^{\prime}-$ bithiophene (1.14 mmol, $190 \mathrm{mg}$ ), and silver nitrate $(161 \mathrm{mg}, 0.95 \mathrm{mmol})$ were reacted in DMSO $(15 \mathrm{~mL})$ according to GP1 to give the desired product. Due to the low solubility of this compound it was not purified by column chromatography but rather by repeated washing of the precipitate with $\mathrm{H}_{2} \mathrm{O}, \mathrm{THF}$, and $\mathrm{CH}_{2} \mathrm{Cl}_{2}$. The product was obtained as a yellow amorphous solid after purification by column chromatography on silica gel using petroleum ether/ethyl acetate

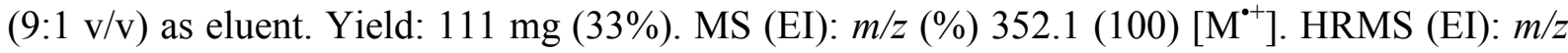
calcd for $\mathrm{C}_{20} \mathrm{H}_{13}{ }^{35} \mathrm{ClS}_{2}: 352.0147$; found: 352.0149 . UV/Vis $\left(\mathrm{CH}_{2} \mathrm{Cl}_{2}\right): \lambda_{\max } 234 \mathrm{~nm}$. Fluorescence $\left(\mathrm{CH}_{2} \mathrm{Cl}_{2}\right): \lambda_{\max } 440 \mathrm{~nm}$.

2-(Biphenyl-4-yl)-thiophene-5-carbonitrile (11). Silver carbonate (494 mg, $1.79 \mathrm{mmol}$ ), [Pd(dppf)Cl $]$ (29 mg, $0.04 \mathrm{mmol}), \mathrm{PPh}_{3}(24 \mathrm{mg}, 0.09 \mathrm{mmol})$, 4-iodobiphenyl (300 mg, 1.07 $\mathrm{mmol})$, and 2-cyanothiophene $(97 \mathrm{mg}, 0.89 \mathrm{mmol}$ ) were reacted according to GP2 to give the desired product as a yellow amorphous solid after purification by column chromatography on silica gel using petroleum ether/ethyl acetate $(9: 1 \mathrm{v} / \mathrm{v})$ as eluent. Yield: $172 \mathrm{mg}(74 \%) .{ }^{1} \mathrm{H}-\mathrm{NMR}$ $\left(400 \mathrm{MHz}, \mathrm{CDCl}_{3}\right): \delta$ 7.61-7.73 (m, $\left.6 \mathrm{H}\right), 7.60(\mathrm{~d}, 1 \mathrm{H}, J 3.9 \mathrm{~Hz}), 7.44-7.51(\mathrm{~m}, 2 \mathrm{H}), 7.36-7.42$ $(\mathrm{m}, 1 \mathrm{H}), 7.31(\mathrm{~d}, 1 \mathrm{H}, J 3.9 \mathrm{~Hz}) \mathrm{ppm} .{ }^{13} \mathrm{C}-\mathrm{NMR}\left(100 \mathrm{MHz}, \mathrm{CDCl}_{3}\right): \delta 151.5,142.3,139.9$, 131.2 , 128.9, 127.9, 127.9, 127.0, 126.8, 123.2, 114.4, 108.2 ppm. MS (EI): $m / z(\%) 261.1$ (100) 
$\left[\mathrm{M}^{\bullet+}\right.$ ]. HRMS (EI): $\mathrm{m} / z$ calcd for $\mathrm{C}_{17} \mathrm{H}_{11} \mathrm{NS}: 261.0612$; found: 261.0614. Anal. calcd for $\mathrm{C}_{17} \mathrm{H}_{11} \mathrm{NS} \cdot 1 / 2 \mathrm{C}_{4} \mathrm{H}_{8} \mathrm{O}_{2}$ : C: $74.72 ; \mathrm{H}: 4.95 ; \mathrm{N}: 4.59 ; \mathrm{S}: 10.50$; found: $\mathrm{C}: 75.13 ; \mathrm{H}: 4.99 ; \mathrm{N}: 4.77$; $\mathrm{S}: 11.02$. UV/Vis $\left(\mathrm{CH}_{2} \mathrm{Cl}_{2}\right): \lambda_{\text {max }} 234 \mathrm{~nm}$. Fluorescence $\left(\mathrm{CH}_{2} \mathrm{Cl}_{2}\right): \lambda_{\max } 393 \mathrm{~nm}$.

2-Bromo-5-(4'-chlorobiphenyl-4-yl)-thiophene (12). Silver carbonate (439 mg, $1.59 \mathrm{mmol}$ ), [Pd(dppf)Cl $\mathrm{Cl}_{2}$ (29 mg, $\left.0.04 \mathrm{mmol}\right), \mathrm{PPh}_{3}(21 \mathrm{mg}, 0.08 \mathrm{mmol})$, 4-chloro-4'-iodobiphenyl (300 $\mathrm{mg}, 0.95 \mathrm{mmol})$, and 2-bromothiophene $(129 \mathrm{mg}, 0.79 \mathrm{mmol})$ were reacted according to $\mathrm{GP} 2$ to give the desired product as a yellow amorphous solid after purification by column chromatography on silica gel using petroleum ether/ethyl acetate $(30: 1 \mathrm{v} / \mathrm{v})$ as eluent. Yield: 215 mg (78\%). ${ }^{1} \mathrm{H}-\mathrm{NMR}\left(400 \mathrm{MHz}, \mathrm{CDCl}_{3}\right): \delta 7.58$ (d, $\left.2 \mathrm{H}, J 8.8 \mathrm{~Hz}\right), 7.55$ (d, $\left.2 \mathrm{H}, J 8.9 \mathrm{~Hz}\right), 7.52$ $(\mathrm{d}, 2 \mathrm{H}, J 8.7 \mathrm{~Hz}), 7.4(\mathrm{~d}, 2 \mathrm{H}, J 8.7 \mathrm{~Hz}), 7.09(\mathrm{~d}, 1 \mathrm{H}, J 3.9 \mathrm{~Hz}), 7.04(\mathrm{~d}, 1 \mathrm{H}, J 3.9 \mathrm{~Hz}) \mathrm{ppm}$. ${ }^{13} \mathrm{C}-\mathrm{NMR}\left(100 \mathrm{MHz}, \mathrm{CDCl}_{3}\right): \delta 145.4,139.5,138.9,133.8,133.1,131.1,128.2,128.3,127.6$, 126.2, 123.6, 111.8 ppm. MS (EI): $m / z$ (\%) 350.0 (100) $\left[\mathrm{M}^{\circ+}\right]$. HRMS (EI): $\mathrm{m} / z$ calcd for $\mathrm{C}_{16} \mathrm{H}_{10}{ }^{79} \mathrm{Br}^{35} \mathrm{ClS}$ : 347.9375; found: 347.9374. Anal. calcd for $\mathrm{C}_{16} \mathrm{H}_{10} \mathrm{BrClS}$ : C: 54.96; H: 2.88; S: 9.17; found: C: 54.69; H: 3.07; S: 9.21. UV/Vis $\left(\mathrm{CH}_{2} \mathrm{Cl}_{2}\right): \lambda_{\max } 234 \mathrm{~nm}$. Fluorescence $\left(\mathrm{CH}_{2} \mathrm{Cl}_{2}\right)$ : $\lambda_{\max } 379 \mathrm{~nm}$.

5-(4'-Chlorobiphenyl-4-yl)-thiophene-2-carbonitrile (13). Silver carbonate (439 mg, 1.59 $\mathrm{mmol}),\left[\mathrm{Pd}(\mathrm{dppf}) \mathrm{Cl}_{2}\right]$ (29 mg, $\left.0.04 \mathrm{mmol}\right), \mathrm{PPh}_{3}(21 \mathrm{mg}, 0.08 \mathrm{mmol})$, 4-chloro-4'-iodobiphenyl (300 mg, $0.95 \mathrm{mmol}$ ), and 2-cyanothiophene ( $86 \mathrm{mg}, 0.79 \mathrm{mmol}$ ) were reacted according to GP2 to give the desired product as a yellow amorphous solid after purification by column chromatography on silica gel using petroleum ether/ethyl acetate $(9: 1 \mathrm{v} / \mathrm{v})$ as eluent. Yield: 175 mg (75\%). ${ }^{1} \mathrm{H}-\mathrm{NMR}\left(400 \mathrm{MHz}, \mathrm{CDCl}_{3}\right): \delta 7.66$ (d, $\left.2 \mathrm{H}, J 10.3 \mathrm{~Hz}\right), 7.60$ (d, $\left.2 \mathrm{H}, J 10.2 \mathrm{~Hz}\right)$, $7.60(\mathrm{~d}, 1 \mathrm{H}, J 4.0 \mathrm{~Hz}), 7.52(\mathrm{~d}, 2 \mathrm{H}, J 8.7 \mathrm{~Hz}), 7.42(\mathrm{~d}, 2 \mathrm{H}, J 8.7 \mathrm{~Hz}), 7.31(\mathrm{~d}, 1 \mathrm{H}, J 3.9 \mathrm{~Hz})$ ppm. ${ }^{13} \mathrm{C}-\mathrm{NMR}\left(100 \mathrm{MHz}, \mathrm{CDCl}_{3}\right): \delta 151.3,141.1,138.6,138.5,134.2,131.7,129.3,128.4$,

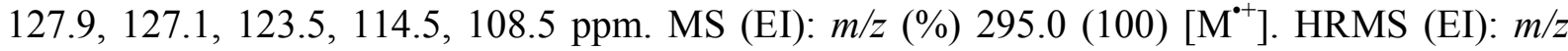
calcd for $\mathrm{C}_{17} \mathrm{H}_{10}{ }^{35} \mathrm{ClNS}: 295.0222$; found: 295.0224 . Anal. calcd for $\mathrm{C}_{17} \mathrm{H}_{10} \mathrm{ClNS} \cdot{ }_{1 / 3} \mathrm{C}_{4} \mathrm{H}_{8} \mathrm{O}_{2}$ : C: 67.72; H: 3.93; N: 4.31; S: 9.86; found: $\mathrm{C}: 67.55 ; \mathrm{H}: 3.81 ; \mathrm{N}: 4.42 ; \mathrm{S}: 9.74$. UV/Vis $\left(\mathrm{CH}_{2} \mathrm{Cl}_{2}\right)$ : $\lambda_{\max } 234 \mathrm{~nm}$. Fluorescence $\left(\mathrm{CH}_{2} \mathrm{Cl}_{2}\right): \lambda_{\max } 398 \mathrm{~nm}$.

5-(4'-Methoxybiphenyl-4-yl)-thiophene-2-carbonitrile (14). Silver carbonate (447 mg, 1.62 mmol), [Pd(dppf)Cl $\mathrm{Cl}_{2}$ (29 mg, $\left.0.04 \mathrm{mmol}\right), \mathrm{PPh}_{3}(22 \mathrm{mg}, 0.08 \mathrm{mmol})$, 4-iodo-4'methoxybiphenyl $(300 \mathrm{mg}, 0.97 \mathrm{mmol})$, and 2-cyanothiophene $(88 \mathrm{mg}, 0.81 \mathrm{mmol})$ reacted according to GP2 to give the desired product as a yellow amorphous solid after purification by column chromatography on silica gel using petroleum ether/ethyl acetate $(5: 1 \mathrm{v} / \mathrm{v})$ as eluent. Yield: $198 \mathrm{mg}(84 \%) .{ }^{1} \mathrm{H}-\mathrm{NMR}\left(400 \mathrm{MHz}, \mathrm{CDCl}_{3}\right.$ ): $\delta 7.69$ (d, $2 \mathrm{H}, J 8.9 \mathrm{~Hz}$ ), 7.63 (d, $2 \mathrm{H}, J 8.9$ Hz), 7.62 (d, 1 H, J 4.0 Hz), 7.57 (d, 2 H, J 8.9 Hz), 7.34 (d, 1 H, J 4.0 Hz), 6.98 (d, 2 H, J 8.9 $\mathrm{Hz}), 3.85(\mathrm{~s}, 3 \mathrm{H}) \mathrm{ppm} .{ }^{13} \mathrm{C}-\mathrm{NMR}\left(100 \mathrm{MHz}, \mathrm{CDCl}_{3}\right): \delta 160.1,151.9,142.1,139.0,132.5,130.1$, 128.3, 127.6, 127.1, 123.5, 114.8, 114.7, 108.3, 55.7 ppm. MS (EI): $m / z(\%) 291.0(100)\left[\mathrm{M}^{*+}\right]$. HRMS (EI): $m / z$ calcd for $\mathrm{C}_{18} \mathrm{H}_{13} \mathrm{NOS}: 291.0718$; found: 291.0719. Anal. calcd for $\mathrm{C}_{18} \mathrm{H}_{13} \mathrm{NOS}$. $1 / 3 \mathrm{C}_{4} \mathrm{H}_{8} \mathrm{O}_{2}$ : C: 72.40; H: 4.92; N: 4.37; S:10.0; found: C: 71.03; H: 5.02; N: 4.40; S: 9.84. $\mathrm{UV} / \mathrm{V}$ is $\left(\mathrm{CH}_{2} \mathrm{Cl}_{2}\right): \lambda_{\max } 234 \mathrm{~nm}$. Fluorescence $\left(\mathrm{CH}_{2} \mathrm{Cl}_{2}\right): \lambda_{\max } 427 \mathrm{~nm}$. 
2-(4'-Methoxybiphenyl-4-yl)-5-nitrothiophene (15). Silver carbonate (444 mg, $1.61 \mathrm{mmol}$ ), [Pd(dppf)Cl $]$ (29 mg, $0.04 \mathrm{mmol}), \mathrm{PPh}_{3}(21 \mathrm{mg}, 0.08 \mathrm{mmol}), 4$-iodo-4'-methoxybiphenyl (300 $\mathrm{mg}, 0.97 \mathrm{mmol})$, and 2-nitrothiophene $(104 \mathrm{mg}, 0.81 \mathrm{mmol})$ reacted according to GP2 to give the desired product as an orange amorphous solid after purification by column chromatography on silica gel using petroleum ether/ethyl acetate (5:1 v/v) as eluent. Yield: $207 \mathrm{mg}(82 \%) .{ }^{1} \mathrm{H}-\mathrm{NMR}$ (400 MHz, $\mathrm{CDCl}_{3}$ ): $\delta 7.92(\mathrm{~d}, 1 \mathrm{H}, J 4.3 \mathrm{~Hz}), 7.67$ (d, $\left.2 \mathrm{H}, J 8.7 \mathrm{~Hz}\right), 7.63$ (d, $\left.2 \mathrm{H}, J 8.8 \mathrm{~Hz}\right)$, $7.55(\mathrm{~d}, 2 \mathrm{H}, J 8.9 \mathrm{~Hz}), 7.26(\mathrm{~d}, 1 \mathrm{H}, J 4.1 \mathrm{~Hz}), 7.00(\mathrm{~d}, 2 \mathrm{H}, J 8.8 \mathrm{~Hz}), 3.87$ (s, $3 \mathrm{H}) \mathrm{ppm} .{ }^{13} \mathrm{C}-$ NMR (100 MHz, $\left.\mathrm{CDCl}_{3}\right): \delta 159.8,152.1,142.7,132.3,130.5,130.0,128.4,128.2,127.6,126.7$, 126.1, 114.6, 55.5 ppm. MS (EI): $m / z$ (\%) $311.1(100)\left[\mathrm{M}^{*+}\right]$. HRMS (EI): $m / z$ calcd for $\mathrm{C}_{17} \mathrm{H}_{13} \mathrm{NO}_{3} \mathrm{~S}$ : 311.0616; found: 311.0617. UV/Vis $\left(\mathrm{CH}_{2} \mathrm{Cl}_{2}\right): \lambda_{\max } 234 \mathrm{~nm}$. Fluorescence $\left(\mathrm{CH}_{2} \mathrm{Cl}_{2}\right): \lambda_{\max } 575 \mathrm{~nm}$.

4'-(5-Methoxy-2-thienyl)-biphenyl-4-carbonitrile (16). Silver carbonate (453 mg, $1.64 \mathrm{mmol}$ ), [Pd(dppf)Cl $\mathrm{Cl}_{2}$ (34 mg, $\left.0.04 \mathrm{mmol}\right), \mathrm{PPh}_{3}(22 \mathrm{mg}, 0.08 \mathrm{mmol}), 4$-iodo-4'-cyanobiphenyl (300 mg, $0.98 \mathrm{mmol}$ ), and 2-methoxythiophene ( $94 \mathrm{mg}, 0.82 \mathrm{mmol}$ ) were reacted according to GP2 to give the desired product as a yellow amorphous solid after purification by column chromatography on silica gel using petroleum ether/ethyl acetate $(9: 1 \mathrm{v} / \mathrm{v})$ as eluent. Yield: $139 \mathrm{mg}(58 \%) .{ }^{1} \mathrm{H}-\mathrm{NMR}$ $\left(400 \mathrm{MHz}, \mathrm{CDCl}_{3}\right): \delta 7.71(\mathrm{~d}, 2 \mathrm{H}, J 8.8 \mathrm{~Hz}), 7.67(\mathrm{~d}, 2 \mathrm{H}, J 8.8 \mathrm{~Hz}), 7.57$ (apparent s, $\left.4 \mathrm{H}\right), 7.03$ $(\mathrm{d}, 1 \mathrm{H}, J 4.0 \mathrm{~Hz}), 6.21(\mathrm{~d}, 1 \mathrm{H}, J 4.0 \mathrm{~Hz}), 3.94(\mathrm{~s}, 3 \mathrm{H}) \mathrm{ppm} .{ }^{13} \mathrm{C}-\mathrm{NMR}\left(100 \mathrm{MHz}, \mathrm{CDCl}_{3}\right): \delta$ $166.7,145.1,137.1,135.3,132.8,129.3,127.7,127.4,125.5,121.5,119.1,110.9,105.1,60.4$ ppm. MS (EI): $m / z(\%) 305.0$ (100) $\left[\mathrm{M}^{\circ+}\right]$. HRMS (EI): $\mathrm{m} / z$ calcd for $\mathrm{C}_{18} \mathrm{H}_{13} \mathrm{NOS}$ : 291.0718; found: 291.0719. UV/Vis $\left(\mathrm{CH}_{2} \mathrm{Cl}_{2}\right): \lambda_{\max } 234 \mathrm{~nm}$. Fluorescence $\left(\mathrm{CH}_{2} \mathrm{Cl}_{2}\right): \lambda_{\max } 448 \mathrm{~nm}$.

5-(4'-Cyanobiphenyl-4-yl)-thiophene-2-carbonitrile (17). Silver carbonate (447 mg, 1.62 mmol), [Pd(dppf)Cl $]$ (30 mg, $0.04 \mathrm{mmol}), \mathrm{PPh}_{3}$ (22 mg, $\left.0.08 \mathrm{mmol}\right)$, 4-iodo-4'-cyanobiphenyl (300 mg, $0.99 \mathrm{mmol}$ ), and 2-cyanothiophene $(89 \mathrm{mg}, 0.82 \mathrm{mmol}$ ) were reacted according to GP2 to give the desired product as a yellow amorphous solid after purification by column chromatography on silica gel using petroleum ether/ethyl acetate $(9: 1 \mathrm{v} / \mathrm{v})$ as eluent. Yield: 124 mg (53\%). ${ }^{1} \mathrm{H}-\mathrm{NMR}\left(400 \mathrm{MHz}, \mathrm{CDCl}_{3}\right): \delta 7.75$ (d, $\left.2 \mathrm{H}, J 8.6 \mathrm{~Hz}\right), 7.71$ (d, $\left.2 \mathrm{H}, J 8.6 \mathrm{~Hz}\right), 7.70$ $(\mathrm{d}, 2 \mathrm{H}, J 8.6 \mathrm{~Hz}), 7.65(\mathrm{~d}, 2 \mathrm{H}, J 8.6 \mathrm{~Hz}), 7.62(\mathrm{~d}, 1 \mathrm{H}, J 3.9 \mathrm{~Hz}), 7.34$ (d, $1 \mathrm{H}, J 3.9 \mathrm{~Hz}) \mathrm{ppm}$. ${ }^{13} \mathrm{C}-\mathrm{NMR}\left(100 \mathrm{MHz}, \mathrm{CDCl}_{3}\right): \delta 144.6,140.4,139.0,133.1,133.0,128.4,128.1,128.0,127.4$,

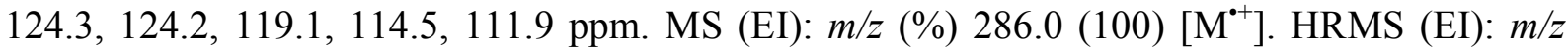
calcd for $\mathrm{C}_{18} \mathrm{H}_{10} \mathrm{~N}_{2} \mathrm{~S}$ : 286.0565; found: 286.0564. UV/Vis $\left(\mathrm{CH}_{2} \mathrm{Cl}_{2}\right): \lambda_{\max } 234 \mathrm{~nm}$. Fluorescence $\left(\mathrm{CH}_{2} \mathrm{Cl}_{2}\right): \lambda_{\max } 411 \mathrm{~nm}$.

\section{Acknowledgements}

Financial support from the DFG is gratefully acknowledged. 


\section{References}

1. Schwab, P. F. H.; Levin, M. D.; Michl, J. Chem. Rev. 1999, 99, 1863.

http://dx.doi.org/10.1021/cr970070x

2. Schwab, P. F. H.; Levin, M. D.; Michl, J. Chem. Rev. 2005, 105, 1197. http://dx.doi.org/10.1021/cr040707u

3. Müllen, K.; Wegner, G. Electronic Materials: The Oligomer Approach; Wiley-VCH: Weinheim, 1999.

4. Nalva, H. S. Handbook of Advanced Electronic and Photonic Materials and Devices; Academic Press: San Diego, 2000.

5. Klauk, H. Organic Electronics- An Industrial Perspective; Wiley-VCH: Weinheim, 2006.

6. Müller, T. J. J.; Bunz, U. H. F. Functional Organic Materials; Wiley-VCH: Weinheim, 2007.

7. Allard, S.; Forster, M.; Souharce, B.; Thiem, H.; Scherf, U. Angew. Chem. 2008, 120, 4138; Angew. Chem. Int. Ed. 2008, 47, 4070.

http://dx.doi.org/10.1002/anie.200701920

8. Mishra, A.;Ma, C.-Q.; Bäuerle, P. Chem. Rev. 2009, 109, 1141. http://dx.doi.org/10.1021/cr8004229

9. Usta, H.; Facchetti, A.; Marks, T. J. Acc. Chem. Res. 2011, 44, 501. http://dx.doi.org/10.1021/ar200006r

10. Mishra, A.; Bäuerle, P. Angew. Chem. 2012, 124, 2060; Angew. Chem. Int. Ed. 2012, 51, 2020.

http://dx.doi.org/10.1002/anie.201102326

11. Chen, Y.; Wan. X.; Long, G. Acc. Chem. Res. 2013, 46, 2645. http://dx.doi.org/10.1021/ar400088c

12. Zhang, L.; Colella, N. S.; Cherniawski, B. P.; Mannsfeld, S. C. B.; Briseno, A. L. ACS Appl. Mater. Interfaces 2014, 6, 5327. http://dx.doi.org/10.1021/am4060468

13. Al-Shamery, K.; Rubahn, H.-G.; Sitter, H. Organic Nanostructures for Next Generation Devices; Springer: Berlin, 2008.

http://dx.doi.org/10.1007/978-3-540-71923-6

14. Schiek, M.; Balzer, F.; Al-Shamery, K.; Lützen, A.; Rubahn, H.-G. Soft Matter 2008, 4, 277. http://dx.doi.org/10.1039/b713295f

15. Schiek, M.; Balzer, F.; Al-Shamery, K.; Brewer, J. R.; Lützen, A.; Rubahn, H.-G. Small 2008, 4, 176.

http://dx.doi.org/10.1002/smll.200700483

16. Steinhart, M.; Wehrspohn, R. B.; Gösele, U.; Wendorff, J. H. Angew. Chem. 2004, 116, 1356; Angew. Chem. Int. Ed. 2004, 43, 1334.

http://dx.doi.org/10.1002/anie.200300614 
17. Nagawa, M.; Hibino, R.; Hotta, S.; Yanagi, H.; Ichikawa, M.; Koyama, T.; Taniguchi, Y. Appl. Phys. Lett. 2002, 80, 544.

http://dx.doi.org/10.1063/1.1435797

18. Yanagi, H.; Morikawa, T.; Hotta, S. Appl. Phys. Lett. 2002, 81, 1512.

http://dx.doi.org/10.1063/1.1502023

19. Ichikawa, M.; Hibino, R.; Inoue, M.; Haitani, T.; Hotta, S.; Koyama, T.; Taniguchi, Y. Adv. Mater. 2003, 15, 213.

http://dx.doi.org/10.1002/adma.200390048

20. Yanagi, H.; Yoshiki, A.; Hotta, S.; Kobayashi, S. J. Appl. Phys. 2004, 96, 4240.

http://dx.doi.org/10.1063/1.1787136

21. Ichikawa, M.; Hibino, R.; Inoue, M.; Haritani, T.; Hotta, S.; Araki, K.-i.; Koyama, T.; Taniguchi, Y. Adv. Mater. 2005, 17, 2073.

http://dx.doi.org/10.1002/adma.200500734

22. Kanazawa, S.; Ichikawa, M.; Koyama, T.; Taniguchi, Y. ChemPhysChem 2006, 7, 1881. http://dx.doi.org/10.1002/cphc.200500669

23. Bando, K.; Nakamura, T.; Masumoto, Y.; Hotta, S. J. Appl. Phys. 2006, 99, 013518. http://dx.doi.org/10.1063/1.2159083

24. Tian, H.; Shi, J.; Yan, D.; Wang, L.; Geng, Y.; Wang, F. Adv. Mater. 2006, 18, 2149. http://dx.doi.org/10.1002/adma.200600178

25. Fujiwara, S.; Bando, K.; Masumoto,Y.; Sasaki, F.; Kobayashi, S.; Haraichi, S.; Hotta, S.; Appl. Phys. Lett. 2007, 91, 021104.

http://dx.doi.org/10.1063/1.2755925

26. Sasaki, F.; Kobayashi, S.; Haraichi, S.; Fujiwara, S.; Bando, K.; Masumoto, Y.; Hotta, S. Adv. Mater. 2007, 19, 3653.

http://dx.doi.org/10.02/adma.200701008

27. Kanazawa, S.; Ichikawa, M.; Fujita, Y.; Koike, R.; Koyama, T.; Taniguchi, Y. Org. Electr. 2008, 9, 425 .

http://dx.doi.org/10.1016/j.orgel.2008.01.006

28. Schiek, M.; Balzer, F.; Al-Shamery, K.; Lützen, A.; Rubahn, H.-G. J. Phys. Chem. C 2009, 113,9601 .

29. de Meijere, A.; Diederich, F. Metal-Catalyzed Cross-Coupling Reactions; $2^{\text {nd }}$ ed., WileyVCH: Weinheim, 2004.

http://dx.doi.org/10.1002/9783527619535

30. Tamao, K.; Kodama, S.; Nakajima, I.; Kumada, M.; Minato, A.; Suzuki, K. Tetrahedron 1982, 38, 3347.

http://dx.doi.org/10.1016/0040-4020(82)80117-8

31. Hotta, S.; Lee, S. A.; Tamaki, T. J. Heterocyclic Chem. 2000, 37, 25.

http://dx.doi.org/10.1002/jhet.5570370105

32. Hotta, S.; Kimura, H.; Lee, S. A.; Tamaki, T. J. Heterocyclic Chem. 2000, 37, 281. http://dx.doi.org/10.1002/jhet.5570370210 
33. Hotta, S. J. Heterocyclic Chem. 2001, 38, 923.

http://dx.doi.org/10.1002/jhet.5570380417

34. Hotta, S.; Katagiri, T. J. Heterocyclic Chem. 2003, 40, 845.

http://dx.doi.org/10.1002/jhet.5570400515

35. Surry, D. S.; Buchwald, S. L. Angew. Chem. 2008, 120, 6438; Angew. Chem. Int. Ed. 2008, 47, 6338 .

http://dx.doi.org/10.1002/anie.200800497

36. Campeau, L.-C.; Fagnou, Chem. Commun. 2006, 1253.

http://dx.doi.org/10.1039/b515481m

37. Alberico, D.; Scott, M. E.; Lautens, M. Chem. Rev. 2007, 107, 174. http://dx.doi.org/10.1021/cr0509760

38. Schiek, M.; Al-Shamery, K.; Lützen, A. Synthesis 2007, 613.

http://dx.doi.org/10.1055/s-2007-965891

39. Wallmann, I.; Schiek, M.; Koch, R.; Lützen, A. Synthesis 2008, 2446.

http://dx.doi.org/10.1055/s-2008-1067163

40. Lavenot, L.; Gozzi, C.; Ilg, K.; Orlova, I.; Penalva, V.; Lemaire, M. J. Organomet. Chem. 1998, 567, 49.

http://dx.doi.org/10.1016/S0022-328X(98)00667-6

41. Jeffery, T. Tetrahedron Lett. 1985, 26, 2667. http://dx.doi.org/10.1016/S0040-4039(00)98131-0

42. Kobayashi, K.; Sugie, A.; Takahashi, M.; Masui, K.; Mori, A. Org. Lett. 2005, 7, 5083. http://dx.doi.org/10.1021/o1052063y

43. Takahashi, M.; Masui, K.; Sekiguchi, H.; Kobayashi, N.; Mori, A.; Funahashi, M.; Tamaoki, N. J. Am. Chem. Soc. 2006, 128, 10930. http://dx.doi.org/10.1021/ja060749v

44. Masuda, N.; Tanba, S.; Sugie, A.; Monguchi, D.; Koumura, N.; Hara, K.; Mori, A. Org. Lett. 2009, 11, 2297.

http://dx.doi.org/10.1021/o1900622h

45. Turner, G. L.; Morris, J. A.; Greaney, M. F. Angew. Chem. 2007, 119, 8142; Angew. Chem. Int. Ed. 2007, 46, 7996. http://dx.doi.org/10.1002/anie.200702141

46. Thiemann, F.; Piehler, T.; Haase, D. Saak, W.; Lützen, A. Eur. J. Org. Chem. 2005, 1991. http://dx.doi.org/10.1002/ejoc.200400796 